\title{
On the self-intersections of curves deep in the lower central series of a surface group
}

\author{
Justin Malestein, Andrew Putman
}

November 30, 2009

\begin{abstract}
We give various estimates of the minimal number of self-intersections of a nontrivial element of the $k^{\text {th }}$ term of the lower central series and derived series of the fundamental group of a surface. As an application, we obtain a new topological proof of the fact that free groups and fundamental groups of closed surfaces are residually nilpotent. Along the way, we prove that a nontrivial element of the $k^{\text {th }}$ term of the lower central series of a nonabelian free group has to have word length at least $k$ in a free generating set.
\end{abstract}

\section{Introduction}

Fix an orientable surface $\Sigma$. The goal of this paper is to quantify the extent to which algebraically complicated elements of $\pi_{1}(\Sigma)$ must exhibit topological complexity.

We begin with some definitions. Let $c: S^{1} \rightarrow \Sigma$ be a closed curve. We define the self-intersection number of $c$, denoted $i(c)$, to be minimum over all curves $c^{\prime}$ which are freely homotopic to $c$ of the quantity

$$
\frac{1}{2}\left|\left\{(x, y) \mid x, y \in S^{1}, x \neq y, c^{\prime}(x)=c^{\prime}(y)\right\}\right| .
$$

The factor $1 / 2$ appears because each self-intersection is counted twice. Also, recall that if $G$ is a group, then the lower central series of $G$ is the inductively defined sequence

$$
\gamma_{1}(G)=G \quad \text { and } \quad \gamma_{k+1}(G)=\left[\gamma_{k}(G), G\right] .
$$

For examples of curves in $\gamma_{j}\left(\pi_{1}(\Sigma)\right)$ together with their self-intersection numbers, see Figure 1.

If $\pi_{1}(\Sigma)$ is nonabelian, then it is easy to see that for $k \geq 1$, there exist $x \in \gamma_{k}\left(\pi_{1}(\Sigma)\right)$ with $i(x)$ arbitrarily large. However, a consequence of Theorems 1.1 and 1.3 below is that there do not exist nontrivial $x \in \gamma_{k}\left(\pi_{1}(\Sigma)\right)$ with $i(x)$ arbitrarily small.

To state these theorems, we define

$$
m_{\mathrm{lcs}}(\Sigma, k)=\min \left\{i(x) \mid x \in \gamma_{k}\left(\pi_{1}(\Sigma)\right), x \neq 1\right\} .
$$

Our first result is the following.

Theorem 1.1. Let $\Sigma_{g, b}$ be a orientable genus $g$ surface with $b \geq 1$ boundary components. Assume that $\pi_{1}\left(\Sigma_{g, b}\right)$ is nonabelian. Then for all $k \geq 1$ we have

$$
m_{l c s}\left(\Sigma_{g, b}, k\right) \geq \frac{k}{4 g+b-1}-1 .
$$




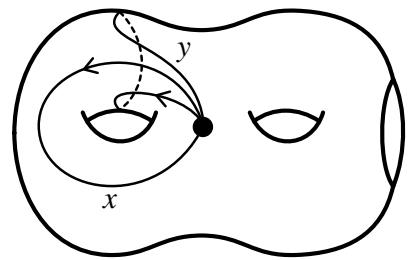

a

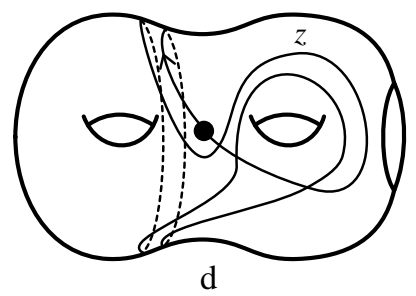

Figure 1: $a, b . z=[x, y] \in \gamma_{2}\left(\pi_{1}(\Sigma)\right)$. Also, $i(z)=0$. $i(z)=3$.

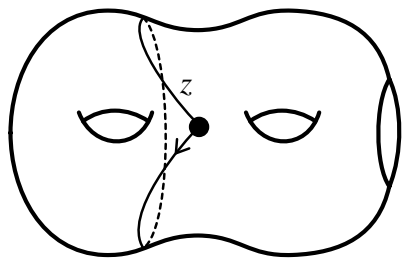

$\mathrm{b}$

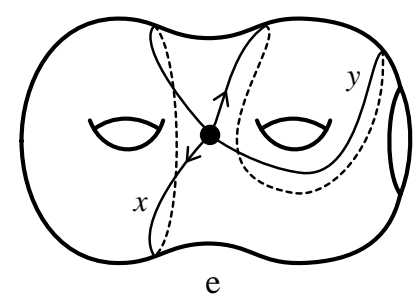

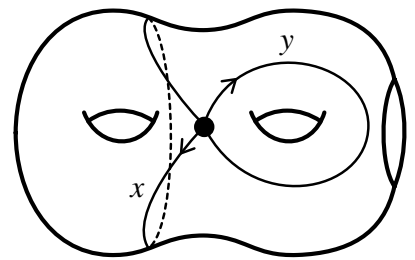

c

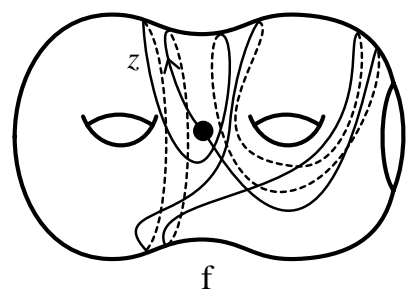

Theorem 1.1 will be proven in $\S 2.1$.

The key to our proof of Theorem 1.1 is the following result, which is proven in $\S 4$. If $G$ is a group and $S \subset G$, then for $x \in\langle S\rangle$ we will denote by $\|x\|_{S}$ the length of the shortest word in $S \cup S^{-1}$ which equals $x$.

Theorem 1.2. Let $F(S)$ be the free group on a set $S$ with $|S|>1$ and let $k \geq 1$. Then for all nontrivial $w \in \gamma_{k}(F(S))$ we have $k \leq\|w\|_{S}$.

This improves upon work of Fox, who in [4, Lemma 4.2] proved a result that implies that $\|w\|_{S} \geq \frac{1}{2} k$. Remark. If we could prove an analogue of Theorem 1.2 for fundamental groups of closed surfaces, then we could also prove an analogue of Theorem 1.1 for closed surfaces.

Remark. We conjecture that Theorem 1.2 is not sharp. Indeed, we suspect that the length of the shortest word in the $k^{\text {th }}$ term of the lower central series of a nonabelian free group is quadratic in $k$. As evidence, in the proofs of the upper bounds of Theorems 1.3 and 1.5 below we will construct elements lying in the $k^{\text {th }}$ term of the lower central series of a rank 2 free group whose word length is quadratic in $k$. If this conjecture were true, then we could replace the lower bound in Theorem 1.1 with a function which is quadratic in $k$.

For general surfaces (not necessarily compact or of finite type), we prove the following.

Theorem 1.3. Let $\Sigma$ be an orientable surface with $\pi_{1}(\Sigma)$ nonabelian. Then for $k \geq 1$ we have

$$
\log _{8}(k)-1 \leq m_{l c s}(\Sigma, k) \leq 8 k^{4} .
$$

The proof of the lower bound in Theorem 1.3 is in $\S 2.3$ and the proof of the upper bound is in $\S 3$.

Remark. Although the lower bound in Theorem 1.3 is weaker than the lower bound in Theorem 1.1 in terms of the order of $k$, it is uniform over all surfaces.

Recall that a group $G$ is residually nilpotent if $\cap_{k=1}^{\infty} \gamma_{k}(G)=1$. Our proof of Theorem 1.2 is an elaboration of a proof due to Fox [4] of a theorem of Magnus [7] that says that free groups are residually nilpotent. Conversely, an immediate consequence of Theorem 1.3 (which does not use Theorem 1.2) is the following theorem, which for surface groups is due independently to Baumslag [2] and Frederick [5]. 
Corollary 1.4. Free groups and fundamental groups of closed surfaces are both residually nilpotent.

Our proof of Theorem 1.3 (and hence of Corollary 1.4) shares some ideas with Hempel's beautiful short proof [6] of the residual finiteness of free groups and surface groups.

The final result of this paper gives an analogue of Theorem 1.3 for the derived series. Recall that if $G$ is a group, then the derived series of $G$ is the inductively defined sequence

$$
G^{(1)}=G \quad \text { and } \quad G^{(k+1)}=\left[G^{(k)}, G^{(k)}\right]
$$

Setting

$$
m_{\mathrm{der}}(\Sigma, k)=\min \left\{i(x) \mid x \in\left(\pi_{1}(\Sigma)\right)^{(k)}, x \neq 1\right\},
$$

our result is as follows.

Theorem 1.5. Let $\Sigma$ be an orientable surface with $\pi_{1}(\Sigma)$ nonabelian. Then for $k \geq 3$ we have

$$
2^{\lceil k / 2\rceil-2} \leq m_{d e r}(\Sigma, k) \leq 2^{4 k-5} .
$$

The lower bound in Theorem 1.5 is proven in $\S 2.4$ and the upper bound is proven in $\S 3$. Our proof of the lower bound in Theorem 1.5 is inspired by an unpublished note of Reznikov [9], which outlines an argument giving a linear lower bound on $m_{\text {der }}(\Sigma, k)$ for $\Sigma$ closed. We remark that though [9] seems to claim that it is dealing with the lower central series, both its definitions and its arguments make it clear that the author intends to discuss the derived series.

Remark. In our definitions above, for $x \in \pi_{1}(\Sigma, *)$ the number $i(x)$ depends only on the free homotopy class of $x$. If we required that our homotopies fix $*$ and $* \in \operatorname{Int}(\Sigma)$, then $i(x)$ would be unchanged. If instead $* \in \partial \Sigma$, then $i(x)$ might differ. However, since the lower central series and derived series are normal, requiring the homotopies to fix the basepoint would not change $m_{\mathrm{lcs}}(\Sigma, k)$ or $m_{\mathrm{der}}(\Sigma, k)$.

Acknowledgments. We would like to thank Khalid Bou-rabee, Nathan Broaddus, Matthew Day, Thomas Koberda, and Ben McReynolds for useful conversations and suggestions. We would especially like to thank Benson Farb for sharing [9] with us and asking whether bounds of the sort we prove might hold.

\section{Lower bounds}

In this section, we prove the lower bounds in Theorems 1.1, 1.3, and 1.5.

\subsection{Lower central series, compact surfaces with boundary}

We begin with Theorem 1.1.

Proof of Theorem 1.1. Let $f: S^{1} \rightarrow \operatorname{Int}\left(\Sigma_{g, b}\right)$ be an immersion whose singularities consist of $i(f)$ isolated double points (see Figure 2.a). Assume that $f$ is freely homotopic to a nontrivial element of $\gamma_{k}\left(\pi_{1}\left(\Sigma_{g, b}\right)\right)$. Our goal is to show that $i(f) \geq \frac{k}{4 g+b-1}-1$.

The first step is to "comb" the double points to a single point on the surface. The immersion $f$ factors through an embedding of a graph whose vertices correspond to the singularities of $f$. More precisely, there is a 4-regular graph $G$ with $i(f)$ vertices, an embedding $\tilde{f}: G \rightarrow \operatorname{Int}\left(\Sigma_{g, b}\right)$, and an 


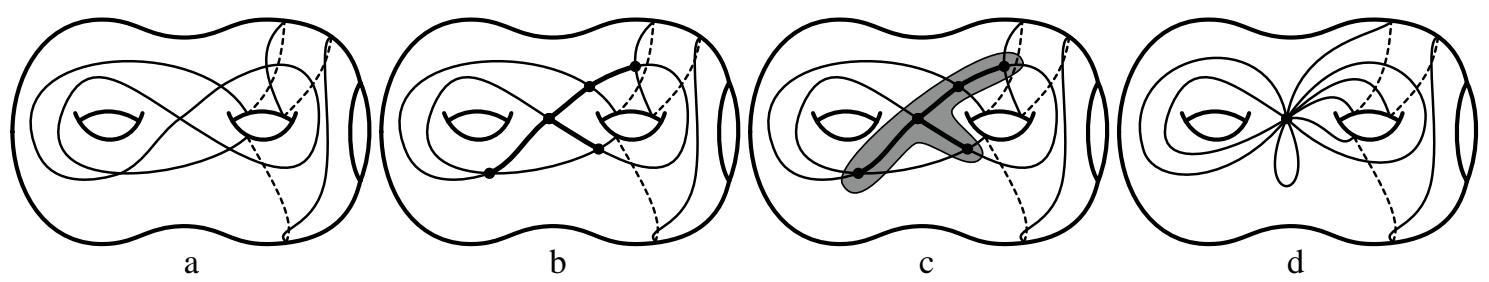

Figure 2: $a$. An immersed curve $f$ whose singularities consist of $i(f)=5$ isolated double points. $\quad b$. The maximal tree $T \quad c$. The 2-disc $D \quad d$. Result of contracting $D$

immersion $c: S^{1} \rightarrow G$ with $f=\tilde{f} \circ c$ such that the inverse image under $c$ of the interior of every edge of $G$ is connected.

Let $T$ be a maximal tree in $G$. Hence $\tilde{f}(T)$ is an embedded tree $\operatorname{in} \operatorname{Int}\left(\Sigma_{g, b}\right)$ (see Figure 2.b). Any sufficiently small closed neighborhood $D$ of $\tilde{f}(T)$ satisfies the following two properties (see Figure 2.c).

- $D$ is homeomorphic to a closed 2-disc.

- For all edges $e$ of $G$ that do not lie in $T$, the set $\tilde{f}(e) \cap D$ has exactly two connected components.

It is easy to see that there is a map $r: \Sigma_{g, b} \rightarrow \Sigma_{g, b}$ such that $r$ is homotopic to the identity, such that $\left.r\right|_{\Sigma_{g, b} \backslash D}$ is injective, and such that $r(D)=*$ for some point $* \in \operatorname{Int}\left(\Sigma_{g, b}\right)$. Let $D^{\prime}=\tilde{f}^{-1}(D)$. By construction, $D^{\prime}$ is a closed regular neighborhood of $T$ in $G$. Set $G^{\prime}=G / D^{\prime}$, so $G^{\prime}$ is a wedge of circles, and let $c^{\prime}: S^{1} \rightarrow G^{\prime}$ be the composition of $c$ with the projection $G \rightarrow G / D^{\prime}$. There is then an embedding $\tilde{f}^{\prime}: G^{\prime} \rightarrow \operatorname{Int}\left(\Sigma_{g, b}\right)$ such that $\tilde{f}^{\prime} \circ c^{\prime}=r \circ f$ (see Figure 2.d).

Let $w \in \pi_{1}\left(\Sigma_{g, b}, *\right)$ be the based curve corresponding to $\tilde{f}^{\prime} \circ c^{\prime}$. Since $\tilde{f}^{\prime} \circ c^{\prime}$ is freely homotopic to $f$, we have $w \in \gamma_{k}\left(\pi_{1}\left(\Sigma_{g, b}, *\right)\right)$. Let $S \subset \pi_{1}\left(\Sigma_{g, b}, *\right)$ be a maximal collection of elements satisfying the following three properties.

- For each circle $L$ in $G^{\prime}$ with $\left.\tilde{f}^{\prime}\right|_{L}$ not null-homotopic, there exists some $x \in S$ such that $\left.\tilde{f}^{\prime}\right|_{L}=$ $x^{ \pm 1}$

- For $x, y \in S$, if $x=y^{ \pm 1}$ then $x=y$.

- The curves in $S$ can be realized simultaneously by simple closed curves that only intersect at *.

Since $G$ is a 4-regular graph with $i(f)$ vertices, it has $2 i(f)$ edges. Also, the maximal tree $T$ has $i(f)$ vertices and hence $i(f)-1$ edges. We conclude that $G^{\prime}$ is a wedge of $2 i(f)-(i(f)-1)=i(f)+1$ circles, so $\|w\|_{S} \leq i(f)+1$.

We will confuse the set of homotopy classes $S$ with the corresponding set of simple closed curves that only intersect at $*$. Via an Euler characteristic calculation, we see that cutting $\Sigma_{g, b}$ along the curves in $S$ yields $b$ annuli and $4 g+b-2$ triangles. By gluing the triangles together in an appropriate manner (as in the standard combinatorial proof of the classification of surfaces; see [8, Chapter 1]), we identify $\Sigma_{g, b}$ with a $(4 g+b)$-sided polygon $P$ with $4 g$ sides identified in pairs, all vertices identified, and annuli glued to the $b$ unpaired sides. Each of the curves in $S$ is identified with either a side of $P$ or an arc in $P$ joining two vertices.

In particular, $S$ contains a free generating set $S^{\prime}$ for $\pi_{1}\left(\Sigma_{g, b}, *\right)$ consisting of the following curves. 
- A curve corresponding to one edge from each of the pairs in the $4 g$ paired edges in $P$.

- A curve corresponding to all but one of the $b$ unpaired edges in $P$.

Observe that every element of $S$ can be written as a word of length at most $4 g+b-1$ in $S^{\prime}$, so $\|w\|_{S^{\prime}} \leq(4 g+b-1)\|w\|_{S}$. Theorem 1.2 says that $k \leq\|w\|_{S^{\prime}}$, so we conclude that

$$
k \leq\|w\|_{S^{\prime}} \leq(4 g+b-1)\|w\|_{S} \leq(4 g+b-1)(i(f)+1) .
$$

Rearranging this inequality gives the desired conclusion.

\subsection{Some preliminary lemmas}

We now prove two lemmas that are needed in the proofs of Theorems 1.3 and 1.5.

Lemma 2.1. Let $\Sigma$ be a compact orientable surface with $\pi_{1}(\Sigma)$ non-abelian and let $f: S^{1} \rightarrow \Sigma$ be a non-nullhomotopic closed curve. Then there exists a degree 8 normal cover $\widetilde{\Sigma} \rightarrow \Sigma$ such that one of the following holds.

- $f$ does not lift to a closed curve on $\widetilde{\Sigma}$.

- $f$ lifts to a closed curve $\tilde{f}: S^{1} \rightarrow \widetilde{\Sigma}$ with $i(\tilde{f})<i(f)$.

Remark. Since the cover in the conclusion of Lemma 2.1 is normal, $f$ lifts to a closed curve if and only if any curve freely homotopic to $f$ lifts to a closed curve.

Proof of Lemma 2.1. By the remark following the lemma, we may assume without loss of generality that $f$ is an immersion whose singularities consist of $i(f)$ isolated double points. There are two cases.

Case 1. $f$ is simple.

We must construct a degree 8 normal cover to which $f$ does not lift to a closed curve. In other words, choosing $* \in f\left(S^{1}\right)$ and letting $x \in \pi_{1}(\Sigma, *)$ be the based curve corresponding to $f$, we must find a finite group $H$ with $|H|=8$ and a surjection $\psi: \pi_{1}(\Sigma, *) \rightarrow H$ with $x \notin \operatorname{ker}(\psi)$.

If $f$ is not nullhomologous and if $\phi: \pi_{1}(\Sigma, *) \rightarrow \mathrm{H}_{1}(\Sigma ; \mathbb{Z})$ is the abelianization map, then $\phi(x)$ is a primitive vector. There is therefore a surjection $\phi^{\prime}: \mathrm{H}_{1}(\Sigma ; \mathbb{Z}) \rightarrow \mathbb{Z} / 8 \mathbb{Z}$ such that $\phi^{\prime}(\phi(x)) \neq 0$. We conclude that we can use $H=\mathbb{Z} / 8 \mathbb{Z}$ and $\psi=\phi^{\prime} \circ \phi$.

Assume now that $f$ is nullhomologous. Letting $g$ be the genus and $b$ the number of boundary components of $\Sigma$, it follows that there is a generating set $S=\left\{\alpha_{1}, \beta_{1}, \ldots, \alpha_{g}, \beta_{g}, x_{1}, \ldots, x_{b}\right\}$ for $\pi_{1}(\Sigma, *)$ such that

$$
\pi_{1}(\Sigma, *)=\left\langle\alpha_{1}, \beta_{1}, \ldots, \alpha_{g}, \beta_{g}, x_{1}, \ldots, x_{b} \mid\left[\alpha_{1}, \beta_{1}\right] \cdots\left[\alpha_{g}, \beta_{g}\right]=x_{1} \cdots x_{b}\right\rangle
$$

and such that $x=\left[\alpha_{1}, \beta_{1}\right] \cdots\left[\alpha_{g^{\prime}}, \beta_{g^{\prime}}\right]$ for some $g^{\prime} \leq g$. Let $H$ be the dihedral group of order 8 , so

$$
H=\left\langle\sigma, r \mid \sigma^{2}=1, r^{4}=1, \sigma r \sigma=r^{-1}\right\rangle .
$$

We define a surjection $\psi: \pi_{1}(\Sigma, *) \rightarrow H$ in the following way. If $b=0$, then $g^{\prime}<g$ and we define $\psi\left(\alpha_{1}\right)=\psi\left(\alpha_{g}\right)=\sigma, \psi\left(\beta_{1}\right)=\psi\left(\beta_{g}\right)=r \sigma$, and $\psi(s)=1$ for all $s \in S$ with $x \notin\left\{\alpha_{1}, \beta_{1}, \alpha_{g}, \beta_{g}\right\}$. It is easy to check that the surface group relation is satisfied and that the resulting homomorphism $\psi$ 

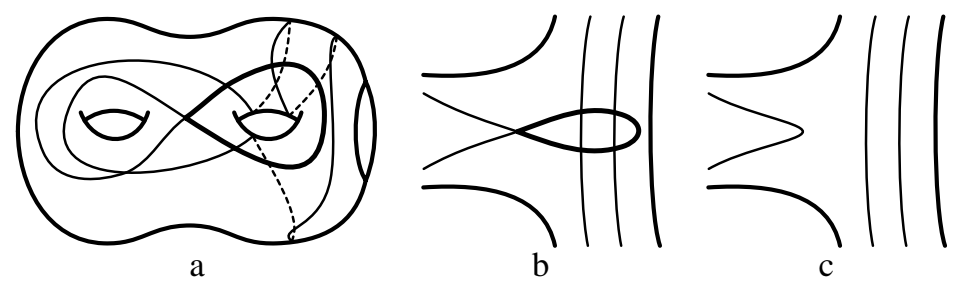

Figure 3: $\quad$ a. A nonsimple closed curve $f$ like in Step 2 of the proof of Lemma 2.1. The simple closed subcurve $f^{\prime}$ is in bold. $\quad$ b. An example of a subcurve $f^{\prime}$ that is nullhomotopic c. We reduce the number of self-intersections of $f$.

is a surjection. If $b>0$, then $\pi_{1}(\Sigma, *)$ is free on $S \backslash\left\{x_{b}\right\}$. We define $\psi\left(\alpha_{1}\right)=\sigma, \psi\left(\beta_{1}\right)=r \sigma$, and $\psi(s)=1$ for all $s \in S \backslash\left\{x_{b}\right\}$ with $s \notin\left\{\alpha_{1}, \beta_{1}, \alpha_{g}, \beta_{g}\right\}$. Trivially $\psi$ extends to a surjection. In either case, we have $\psi(x)=[\sigma, r \sigma] \neq 1$, as desired.

Case 2. $f$ is not simple.

Let $A$ be the set of nontrivial proper subarcs of $S^{1}$ whose endpoints are mapped by $f$ to the same point of $\Sigma$. By assumption $A$ is finite and nonempty. Partially order the elements of $A$ by inclusion and let $\alpha$ be a minimal element with endpoints $a_{1}$ and $a_{2}$. Since $\alpha \in A$, the map $\left.f\right|_{\alpha}: \alpha \rightarrow \Sigma$ factors through a map $f^{\prime}: S^{1} \rightarrow \Sigma$, and from the minimality of $\alpha$ we deduce that $f^{\prime}$ is a simple closed curve (see Figure 3.a). In addition, $f^{\prime}$ is not nullhomotopic, since if $f^{\prime}$ were nullhomotopic then we could homotope $f$ so as to decrease its number of self-intersections (see Figures 3.b-c).

By Case 1 , there is a degree 8 normal cover $\widetilde{\Sigma} \rightarrow \Sigma$ to which $f^{\prime}$ does not lift to a closed curve. If $f$ does not lift to a closed curve on $\widetilde{\Sigma}$, then we are done. Assume, therefore, that $f$ can be lifted to a closed curve $\tilde{f}: S^{1} \rightarrow \widetilde{\Sigma}$. Define

$$
\begin{aligned}
& D(f)=\left\{(x, y) \mid x, y \in S^{1}, x \neq y, f(x)=f(y)\right\}, \\
& D(\tilde{f})=\left\{(x, y) \mid x, y \in S^{1}, x \neq y, \tilde{f}(x)=\tilde{f}(y)\right\} .
\end{aligned}
$$

We clearly have $D(\tilde{f}) \subset D(f)$. Moreover, by construction $\left(a_{1}, a_{2}\right) \notin D(\tilde{f})$. We conclude that $\tilde{f}$ has fewer self-intersections than $f$, so $i(\tilde{f})<i(f)$, as desired.

We will also need the following simple lemma, which allows us to deduce results about noncompact surfaces from results about compact surfaces.

Lemma 2.2. Let $\Sigma$ be an oriented surface with $\pi_{1}(\Sigma)$ nonabelian. Also, let $f: S^{1} \rightarrow \Sigma$ be a nonnullhomotopic closed curve which is freely homotopic to an element of $\gamma_{k}\left(\pi_{1}(\Sigma)\right)$ for some $k \geq 1$. Then there is a compact surface $\Sigma^{\prime}$ with $\pi_{1}\left(\Sigma^{\prime}\right)$ nonabelian and an embedding $i: \Sigma^{\prime} \hookrightarrow \Sigma$ satisfying the following properties.

- There is a map $f^{\prime}: S^{1} \rightarrow \Sigma^{\prime}$ such that $f=i \circ f$.

- The curve $f^{\prime}$ is freely homotopic to an element of $\gamma_{k}\left(\pi_{1}\left(\Sigma^{\prime}\right)\right)$.

Proof. Any iterated commutator only involves a finite number of curves and any homotopy stays within a compact subset of $\Sigma$. 


\subsection{Lower central series, general surfaces}

We now prove the lower bound in Theorem 1.3. The proof will require the following lemma.

Lemma 2.3. Fix $p, n, m \geq 1$ with $p$ prime, and let $G_{0} \triangleright G_{1} \triangleright \cdots \triangleright G_{n}$ be a subnormal sequence of groups with $\left[G_{i-1}: G_{i}\right]=p^{m}$ for $1 \leq i \leq n$. Then there exists some group $H$ such that $H<G_{n}$, such that $H \triangleleft G_{0}$, and such that $\left[G_{0}: H\right]=\bar{p}^{N}$ for some $1 \leq N \leq m \frac{p^{m n}-1}{p^{m}-1}$.

For the proof of Lemma 2.3, we will need the following.

Lemma 2.4. Fix $p, r, s \geq 1$ with $p$ prime, and let $A \triangleright B \triangleright C$ be groups with $[A: B]=p^{r}$ and $[B: C]=$ $p^{s}$. Then there exists a group $D$ such that $D<C$, such that $D \triangleleft A$, and such that $[A: D]=p^{N}$ for some $1 \leq N \leq p^{r} s+r$.

Proof. Define $D=\bigcap_{a \in A} a^{-1} C a$. Clearly we have $D<C$ and $D \triangleleft A$, so we must only prove the indicated result about $[A: D]$. Let $T=\left\{a_{1}, \ldots, a_{p^{r}}\right\}$ be a complete set of coset representatives for $B$ in $A$ with $a_{1}=1$. Hence we have $D=\bigcap_{j=1}^{p^{r}} a_{j}^{-1} C a_{j}$. For $1 \leq i \leq p^{r}$, define $C_{i}=\bigcap_{j=1}^{i} a_{j}^{-1} C a_{j}$. We thus have

$$
A \triangleright B \triangleright C=C_{1} \triangleright C_{2} \triangleright \cdots \triangleright C_{p^{r}}=D .
$$

We claim that for $1<i \leq p^{r}$ we have $\left[C_{i-1}: C_{i}\right]=p^{k_{i}}$ for some $0 \leq k_{i} \leq s$. Indeed, we have

$$
C_{i-1} / C_{i}=C_{i-1} /\left(a_{i}^{-1} C a_{i} \cap C_{i-1}\right) \cong\left(C_{i-1} \cdot\left(a_{i}^{-1} C a_{i}\right)\right) / a_{i}^{-1} C a_{i}<B / a_{i}^{-1} C a_{i} .
$$

Since $\left[B: a_{i}^{-1} C a_{i}\right]=[B: C]=p^{s}$, the claim follows. We conclude that

$$
[A: D]=[A: B][B: C]\left[C_{1}: C_{2}\right] \cdots\left[C_{p^{r}-1}: C_{p^{r}}\right]=p^{r} p^{s} p^{k_{2}} \cdots p^{k^{r}} \leq p^{r}\left(p^{s}\right)^{p^{r}}
$$

as desired.

Proof of Lemma 2.3. The proof will be by induction on $n$. The base case $n=1$ is trivial. Now assume that $n>1$ and that the lemma is true for all smaller $n$. Applying the inductive hypothesis to the sequence $G_{1} \triangleright \cdots \triangleright G_{n}$, we obtain a group $H^{\prime}$ such that $H^{\prime}<G_{n}$, such that $H^{\prime} \triangleleft G_{1}$, and such that $\left[G_{1}: H^{\prime}\right]=p^{N^{\prime}}$ with $N^{\prime} \leq m \frac{p^{m(n-1)}-1}{p^{m}-1}$. We can therefore apply Lemma 2.4 to the sequence $G_{0} \triangleright G_{1} \triangleright H^{\prime}$ and obtain a group $H$ such that $H<H^{\prime}<G_{n}$, such that $H \triangleleft G_{0}$, and such that $\left[G_{0}: H\right]=p^{N}$ for some $N$ that satisfies

$$
N \leq p^{m} N^{\prime}+m \leq p^{m} m \frac{p^{m(n-1)}-1}{p^{m}-1}+m=m \frac{p^{m n}-p^{m}}{p^{m}-1}+m=m \frac{p^{m n}-1}{p^{m}-1},
$$

as desired.

We will also need the following standard property of $p$-groups. Recall that a group $G$ is at most $n$-step nilpotent if $\gamma_{n}(G)=1$.

Lemma 2.5 ([10, Theorem 5.33]). Let $p$ be a prime and let $G$ be a group with $|G|=p^{n}$ for some $n \in \mathbb{N}$. Then $G$ is at most $n$-step nilpotent.

We can now prove the lower bound in Theorem 1.3. 
Proof of Theorem 1.3, lower bound. Let $f: S^{1} \rightarrow \Sigma$ be an immersion whose singularities consist of $i(f)$ isolated double points. Assume that $f$ is freely homotopic to a nontrivial element of $\gamma_{k}\left(\pi_{1}(\Sigma)\right)$. Our goal is to show that $i(f) \geq \log _{8}(k)-1$; i.e. that $k \leq 8^{i(f)+1}$.

By Lemma 2.2, we may assume that $\Sigma$ is compact. Choose a basepoint $* \in f\left(S^{1}\right)$ and let $x \in \pi_{1}(\Sigma, *)$ be the based curve corresponding to $f$. Applying Lemma 2.1 repeatedly, we obtain a subnormal sequence

$$
\pi_{1}(\Sigma, *)=G_{0} \triangleright G_{1} \triangleright \cdots \triangleright G_{n}
$$

with $n \leq i(f)+1$ such that $x \notin G_{n}$ and such that $\left[G_{i-1}: G_{i}\right]=2^{3}$ for $1 \leq i \leq n$. Applying Lemma 2.3 , we obtain a group $H$ such that $H<G_{n}$, such that $H \triangleleft \pi_{1}(\Sigma, *)$, and such that $\left[\pi_{1}(\Sigma, *): H\right]=2^{N}$ for some

$$
N \leq 3 \frac{2^{3 n}-1}{2^{3}-1} \leq 8^{n} \leq 8^{i(f)+1} .
$$

By Lemma 2.5, we deduce that $\pi_{1}(\Sigma, *) / H$ is at most $8^{i(f)+1}$-step nilpotent. In other words,

$$
\gamma_{8^{i(f)+1}}\left(\pi_{1}(\Sigma, *)\right)<H .
$$

Since $H$ is a normal subgroup of $\pi_{1}(\Sigma, *)$ and $f$ is freely homotopic to $x \notin H$, it follows that $f$ is not freely homotopic to any element of $H$. We conclude that $k \leq 8^{i(f)+1}$, as desired.

\subsection{Derived series}

We now prove the lower bound in Theorem 1.5. The proof will require the following lemma.

Lemma 2.6. Let $\Sigma$ be an orientable surface (not necessarily compact) with $\pi_{1}(\Sigma)$ nonabelian. Also, let $f: S^{1} \rightarrow \Sigma$ be a non-nullhomotopic simple closed curve. Then $f$ is not freely homotopic to any element of $\gamma_{3}\left(\pi_{1}(\Sigma)\right)$.

Proof. By Lemma 2.2, we may assume that $\Sigma$ is compact. Assume that $f$ is freely homotopic to $x \in \pi_{1}(\Sigma)$. Since $f$ is simple, Lemma 2.1 implies that there is a finite group $H$ with $|H|=2^{3}$ and a surjection $\psi: \pi_{1}(\Sigma) \rightarrow H$ such that $\psi(x) \neq 1$. Lemma 2.5 says that $H$ is at most 3-step nilpotent, so $\gamma_{3}\left(\pi_{1}(\Sigma)\right) \subset \operatorname{ker}(\psi)$. We conclude that $x \notin \gamma_{3}\left(\pi_{1}(\Sigma)\right)$, as desired.

We will also need the following standard lemma.

Lemma 2.7 ([10, Exercise 5.50]). If $G$ is a group, then for all $k \geq 1$ we have $G^{(k)}<\gamma_{2^{k-1}}(G)$.

We can now prove the lower bound in Theorem 1.5.

Proof of Theorem 1.5, lower bound. We will prove that $2^{\lceil k / 2\rceil-2} \leq m_{\mathrm{der}}(\Sigma, k)$ for $k \geq 3$ by induction on $k$. The base cases $k=3$ and $k=4$ follow from Lemma 2.6 combined with Lemma 2.7. Now assume that $k>4$ and that the result is true for all smaller $k$. It is enough to prove that

$$
m_{\mathrm{der}}(\Sigma, k) \geq 2 \cdot m_{\mathrm{der}}(\Sigma, k-2)
$$

Consider an immersion $f: S^{1} \rightarrow \Sigma$ whose singularities consist of $i(f)$ isolated double points. Assume that $i(f)<2 \cdot m_{\mathrm{der}}(\Sigma, k-2)$. Our goal is to show that $f$ is not freely homotopic to any element of $\left(\pi_{1}(\Sigma)\right)^{(k)}$. 
Let $\pi: \widetilde{\Sigma} \rightarrow \Sigma$ be the normal covering corresponding to the subgroup $\left(\pi_{1}(\Sigma)\right)^{(k-2)}$. If $f$ does not lift to a closed curve in $\widetilde{\Sigma}$, then $f$ is not freely homotopic to any element of $\left(\pi_{1}(\Sigma)\right)^{(k-2)}$, and thus is certainly not freely homotopic to any element of $\left(\pi_{1}(\Sigma)\right)^{(k)}$. Assume, therefore, that there is a lift $\tilde{f}: S^{1} \rightarrow \widetilde{\Sigma}$ of $f$. We claim that $\tilde{f}$ is a simple closed curve. Indeed, define

$$
\begin{aligned}
& D(f)=\left\{(x, y) \mid x, y \in S^{1}, x \neq y, f(x)=f(y)\right\}, \\
& D(\tilde{f})=\left\{(x, y) \mid x, y \in S^{1}, x \neq y, \tilde{f}(x)=\tilde{f}(y)\right\} .
\end{aligned}
$$

Clearly $D(\tilde{f}) \subset D(f)$, and we want to prove that $D(\tilde{f})=\emptyset$. Consider any $(x, y) \in D(f)$. The points $x$ and $y$ divide $S^{1}$ into two arcs $\alpha$ and $\alpha^{\prime}$, and the restrictions of $f$ to both $\alpha$ and $\alpha^{\prime}$ are closed curves. The number of self-intersections of one of $\left.f\right|_{\alpha}$ and $\left.f\right|_{\alpha^{\prime}}$ (say $\left.f\right|_{\alpha}$ ) is less than half of the number of self-intersections of $f$. Hence the closed curve defined by $\left.f\right|_{\alpha}$ has fewer than than $m_{\text {der }}(\Sigma, k-2)$ self-intersections, so it is not freely homotopic to any element of $\left(\pi_{1}(\Sigma)\right)^{(k-2)}$. We conclude that $\left.\tilde{f}\right|_{\alpha}$ is not a closed curve, so $(x, y) \notin D(\tilde{f})$, as desired.

Observe now that by Lemmas 2.6 and 2.7, the curve $\tilde{f}$ is not freely homotopic to any element of $\left(\pi_{1}(\widetilde{\Sigma})\right)^{(3)}$. Since

$$
\left(\pi_{1}(\widetilde{\Sigma})\right)^{(3)}=\left(\left(\pi_{1}(\Sigma)\right)^{(k-2)}\right)^{(3)}=\left(\pi_{1}(\Sigma)\right)^{(k)},
$$

we conclude that $f$ is not freely homotopic to any element of $\left(\pi_{1}(\Sigma)\right)^{(k)}$, as desired.

\section{Upper bounds}

We now prove the upper bounds in Theorems 1.3 and 1.5. We will need two lemmas.

Lemma 3.1. Let $(\Sigma, *)$ be a based surface and let $S \subset \pi_{1}(\Sigma, *)$ be a set consisting of elements that can be realized simultaneously by simple closed curves that only intersect at $*$. Then for all $x \in\langle S\rangle \subset \pi_{1}(\Sigma, *)$, we have $i(x) \leq\left(\begin{array}{c}\|x\|_{2} \\ 2\end{array}\right)$.

Proof. We can assume that $* \in \operatorname{Int}(\Sigma)$. Set $n=\|x\|_{S}$ and write $x=s_{1} \cdots s_{n}$ with $s_{i} \in S \cup S^{-1}$ for $1 \leq i \leq n$. For $1 \leq i \leq n$, we can choose embeddings $f_{i}: S^{1} \rightarrow \Sigma$ such that $f_{i}$ represents $s_{i}$. Moreover, we can choose the $f_{i}$ such that $f_{i}\left(S^{1}\right) \cap f_{j}\left(S^{1}\right)=\{*\}$ for $1 \leq i<j \leq n$. Let $D \subset \Sigma$ be a closed embedded 2-disc with $* \in D$ such that $f_{i}\left(S^{1}\right) \cap D$ is a connected arc for all $1 \leq i \leq n$. Parametrize $D$ such that $D$ is the unit disc in $\mathbb{R}^{2}$ and $*=(0,0)$. For $1 \leq i \leq n$, let $f_{i}^{\prime}:[0,1] \rightarrow \Sigma$ be a parametrization of the oriented arc $f_{i}\left(S^{1}\right) \backslash \operatorname{Int}(D)$. Observe that for $1 \leq i<j \leq n$ we have $f_{i}^{\prime}([0,1]) \cap f_{j}^{\prime}([0,1])=\emptyset$.

We can now construct a curve $f: S^{1} \rightarrow \Sigma$ that is freely homotopic to $x$ in the following way. The curve $f$ first traverses $f_{1}^{\prime}$, then goes along a straight line in $D$ from $f_{1}^{\prime}(1)$ to $f_{2}^{\prime}(0)$, then traverses $f_{2}^{\prime}$, then goes along a straight line in $D$ from $f_{2}^{\prime}(1)$ to $f_{3}^{\prime}(0)$, then traverses $f_{3}^{\prime}$, etc. The curve $f$ ends with a straight line in $D$ from $f_{n}^{\prime}(1)$ to $f_{1}^{\prime}(0)$. Clearly $f$ is freely homotopic to $x$. Moreover, all self-intersections of $f$ must occur in $D$. Since $f\left(S^{1}\right) \cap D$ consists of $n$ straight lines and any two of these lines can intersect at most once, we conclude that $f$ has at most $\left(\begin{array}{l}n \\ 2\end{array}\right)$ self-intersections, as desired.

Lemma 3.2. Let $S=\left\{a_{1}, a_{2}\right\}$ and let $F_{S}$ be the free group on $S$. Then for all $k \geq 1$ there exists some $w \in F_{S}$ with $w \neq 1$ such that $\|w\|_{S} \leq 4^{k-1}$ and $w \in F_{S}^{(k)}$. 
Proof. Define elements $x_{k}$ and $y_{k}$ inductively as follows.

$$
\begin{array}{rll}
x_{1}=a_{1} & \text { and } & y_{1}=a_{2}, \\
x_{k}=\left[x_{k-1}, y_{k-1}\right] & \text { and } & y_{k}=\left[x_{k-1}, y_{k-1}^{-1}\right] .
\end{array}
$$

Clearly $\left\|x_{k}\right\|_{S} \leq 4^{k-1}$ and $x_{k} \in F_{S}^{(k)}$ for $k \geq 1$. We must therefore only prove that $x_{k} \neq 1$ for $k \geq 1$. In fact, we will prove by induction on $k$ that $x_{k}$ and $y_{k}$ generate a rank 2 free subgroup of $F_{S}$ for $k \geq 1$. The base case $k=1$ is trivial. Now assume that $k>1$ and that $x_{k-1}$ and $y_{k-1}$ generate a rank 2 free subgroup. Since neither $x_{k}$ nor $y_{k}$ is trivial, they must generate either a rank 2 or rank 1 free subgroup. But since $x_{k-1}$ and $y_{k-1}$ generate a rank 2 free subgroup, we have

$$
\left[x_{k}, y_{k}\right]=\left[\left[x_{k-1}, y_{k-1}\right],\left[x_{k-1}, y_{k-1}^{-1}\right]\right] \neq 1,
$$

so we conclude that $x_{k}$ and $y_{k}$ cannot generate a rank 1 subgroup.

We can now prove the upper bounds in Theorems 1.3 and 1.5.

Proof of Theorem 1.5, upper bound. We wish to prove that $m_{\mathrm{der}}(\Sigma, k) \leq 2^{4 k-5}$ for $k \geq 3$. In fact, this inequality holds for $k \geq 1$ (the assumption that $k \geq 3$ is necessary only in the lower bound), so fix $k \geq 1$. We claim that there exists some $a_{1}, a_{2} \in \pi_{1}(\Sigma, *)$ that generate a rank 2 free subgroup of $\pi_{1}(\Sigma, *)$ and can be realized simultaneously by simple closed curves that only intersect at $*$. If $\Sigma$ is compact, then this is trivial. Otherwise, $\pi_{1}(\Sigma, *)$ must be a nonabelian free group (see, e.g., [1, $\S 44 \mathrm{~A}])$, so we can find $a_{1}^{\prime}, a_{2}^{\prime} \in \pi_{1}(\Sigma, *)$ that generate a rank 2 free subgroup. Like in the proof of the Theorem 1.1, we can "comb" the intersections and self-intersections of $a_{1}^{\prime}$ and $a_{2}^{\prime}$ to $*$ and find a set $S^{\prime} \subset \pi_{1}(\Sigma, *)$ of elements that can be realized simultaneously by simple closed curves that only intersect at $*$ such that both $a_{1}^{\prime}$ and $a_{2}^{\prime}$ can be expressed as products of elements of $S^{\prime} \cup\left(S^{\prime}\right)^{-1}$. There must then exist $a_{1}, a_{2} \in S^{\prime}$ that generate a rank 2 free subgroup, as desired.

Set $S=\left\{a_{1}, a_{2}\right\}$. By Lemma 3.2, there is some $w \in\langle S\rangle$ such that $\|w\|_{S} \leq 4^{k-1}$ and $w \in$ $\left(\pi_{1}(\Sigma)\right)^{(k)}$. By Lemma 3.1, we deduce that

$$
i(w) \leq\left(\begin{array}{c}
\|x\|_{S} \\
2
\end{array}\right) \leq \frac{4^{k-1}\left(4^{k-1}-1\right)}{2} \leq \frac{1}{2} 4^{2 k-2}=2^{4 k-5},
$$

so $m_{\mathrm{der}}(\Sigma, k) \leq 2^{4 k-5}$, as desired.

Proof of Theorem 1.3, upper bound. Fix $k \geq 1$. We can then find an integer $l$ such that $\log _{2}(k) \leq$ $l-1 \leq \log _{2}(k)+1$. The upper bound of Theorem 1.5 (which as we observed above holds for $k \geq 1$ ) implies that we can find $x \in\left(\pi_{1}(\Sigma)\right)^{(l)}$ such that

$$
i(x) \leq 2^{4 l-5} \leq 2^{4\left(\log _{2}(k)+2\right)-5}=8 k^{4} .
$$

By Lemma 2.7, we have $x \in \gamma_{k}\left(\pi_{1}(\Sigma)\right)$, so we conclude that $m_{\mathrm{lcs}}(\Sigma, k) \leq 8 k^{4}$, as desired. 


\section{Word length in the lower central series}

In this section, we will prove Theorem 1.2. As was indicated in the introduction, this proof is inspired by an argument of Fox [4, Lemma 4.2]. Our main tool will be the Fox free differential calculus, so we begin by recalling a number of basic facts about this calculus. A good reference is [4].

Let $F$ be the free group on a set $S$ and let $\varepsilon: \mathbb{Z} F \rightarrow \mathbb{Z}$ be the augmentation map; i.e. the unique linear map with $\varepsilon(g)=1$ for all $g \in F(S)$.

Definition 4.1. A free derivative is a linear map $D: \mathbb{Z} F \rightarrow \mathbb{Z} F$ such that $D(x y)=(D(x)) \varepsilon(y)+x D(y)$ for all $x, y \in \mathbb{Z} F$.

An easy induction establishes that if $D$ is a free derivative, then for $v_{1}, \ldots, v_{k} \in \mathbb{Z} F$ we have

$$
D\left(v_{1} \cdots v_{k}\right)=\sum_{i=1}^{k}\left(v_{1} \cdots v_{i-1}\right)\left(D\left(v_{i}\right)\right) \varepsilon\left(v_{i+1}\right) \cdots \varepsilon\left(v_{k}\right) .
$$

A consequence of (1) is that for $g \in F$, we have

$$
D\left(g^{-1}\right)=-g^{-1} D(g) .
$$

The basic existence result for free derivatives is the following.

Lemma $4.2([4, \S 2])$. For every $s \in S$, there is a unique free derivative $D_{s}$ satisfying $D_{s}(s)=1$ and $D_{s}\left(s^{\prime}\right)=0$ for $s^{\prime} \in S$ with $s^{\prime} \neq s$.

By (1) and (2), we have

$$
\varepsilon\left(D_{s}\left(s^{k}\right)\right)=k
$$

for all $s \in S$ and $k \in \mathbb{Z}$.

For $k \geq 1$ and $s_{1}, \ldots, s_{k} \in S$, we will call the product $D_{s_{1}} \cdots D_{s_{k}}$ a free derivative of order $k$. The basic fact connecting the Fox free differential calculus to the lower central series of $F$ is the following easy lemma.

Lemma $4.3([3,3.1])$. For $k \geq 2$ and $g \in \gamma_{k}(F)$, we have $\varepsilon(D(g))=0$ for all free derivatives $D$ of order less than or equal to $k-1$.

We can now prove Theorem 1.2.

Proof of Theorem 1.2. Consider $w \in \gamma_{k}(F(S))$ with $w \neq 1$. Our goal is to show that $k \leq\|w\|_{S}$. We will produce a free derivative $D$ whose order is at most $\|w\|_{S}$ such that $\varepsilon(D(w)) \neq 0$. By Lemma 4.3 , it will follow that

$$
w \notin \gamma_{1+\|w\|_{S}}(F(S))
$$

and hence that $k \leq\|w\|_{s}$.

Write $w=u_{1} \cdots u_{n}$ with $u_{i}=s_{i}^{m_{i}}$ for some $s_{i} \in S$ and $m_{i} \in \mathbb{Z} \backslash\{0\}$ for $1 \leq i \leq n$. Choose this expression such that $s_{i} \neq s_{i+1}$ for $1 \leq i<n$. We thus have $n \leq\|w\|_{S}$. Define $D=D_{s_{1}} \cdots D_{s_{n}}$. We must show that $\varepsilon(D(w)) \neq 0$. In fact, we will show that for all $1 \leq j \leq n$ we have

$$
\begin{aligned}
& D_{s_{j}} D_{s_{j+1}} \cdots D_{s_{n}}(w) \\
& =\sum_{1 \leq i_{j}<i_{j+1}<\cdots<i_{n} \leq n}\left(u_{1} \cdots u_{i_{j}-1}\right)\left(D_{s_{j}}\left(u_{i_{j}}\right)\right) \varepsilon\left(D_{s_{j+1}}\left(u_{i_{j+1}}\right)\right) \cdots \varepsilon\left(D_{s_{n}}\left(u_{i_{n}}\right)\right) .
\end{aligned}
$$


In particular, the case $j=1$ will yield

$$
D(w)=D_{s_{1}}\left(u_{1}\right) \varepsilon\left(D_{s_{2}}\left(u_{2}\right)\right) \cdots \varepsilon\left(D_{s_{n}}\left(u_{n}\right)\right) .
$$

Using (3), we will then be able to deduce that

$$
\varepsilon(D(w))=\varepsilon\left(D_{s_{1}}\left(u_{1}\right)\right) \cdots \varepsilon\left(D_{s_{n}}\left(u_{n}\right)\right)=m_{1} \cdots m_{n} \neq 0,
$$

as desired.

The proof of (4) will be by induction on $n-j$. The base case $n-j=0$ follows from (1) and the fact that $\varepsilon\left(u_{i}\right)=1$ for all $1 \leq i \leq n$. Now assume that $n-j>0$ and that (4) holds for all smaller $n-j$. Since $s_{i} \neq s_{i+1}$ for $1 \leq i<n$, we must have $D_{s_{j}} D_{s_{j+1}}\left(u_{j+1}\right)=0$. Using this together with (1), our inductive hypothesis, and the fact that $\varepsilon\left(u_{i}\right)=1$ for all $1 \leq i \leq n$, we obtain

$$
\begin{aligned}
D_{s_{j}} D_{s_{j+1}} \cdots D_{s_{n}}(w) & D_{s_{j}}\left(\sum_{1 \leq i_{j+1}<\cdots<i_{n} \leq n}\left(u_{1} \cdots u_{i_{j+1}-1}\right)\left(D_{s_{j+1}}\left(u_{i_{j+1}}\right)\right) \varepsilon\left(D_{s_{j+2}}\left(u_{i_{j+2}}\right)\right) \cdots \varepsilon\left(D_{s_{n}}\left(u_{i_{n}}\right)\right)\right. \\
& =\sum_{1 \leq i_{j+1}<\cdots<i_{n} \leq n}\left(\sum_{i=1}^{i_{j+1}-1}\left(u_{1} \cdots u_{i-1}\right)\left(D_{s_{j}}\left(u_{i}\right)\right) \varepsilon\left(D_{s_{j+1}}\left(u_{i_{j+1}}\right)\right) \cdots \varepsilon\left(D_{s_{n}}\left(u_{i_{n}}\right)\right)\right) \\
& =\sum_{1 \leq i_{j}<i_{j+1}<\cdots<i_{n} \leq n}\left(u_{1} \cdots u_{i_{j}-1}\right)\left(D_{s_{j}}\left(u_{i_{j}}\right)\right) \varepsilon\left(D_{s_{j+1}}\left(u_{i_{j+1}}\right)\right) \cdots \varepsilon\left(D_{s_{n}}\left(u_{i_{n}}\right)\right),
\end{aligned}
$$

and we are done.

\section{References}

[1] L. V. Ahlfors and L. Sario, Riemann surfaces, Princeton Univ. Press, Princeton, N.J., 1960.

[2] G. Baumslag, On generalised free products, Math. Z. 78 (1962), 423-438.

[3] K.-T. Chen, R. H. Fox and R. C. Lyndon, Free differential calculus. IV. The quotient groups of the lower central series, Ann. of Math. (2) 68 (1958), 81-95.

[4] R. H. Fox, Free differential calculus. I. Derivation in the free group ring, Ann. of Math. (2) 57 (1953), $547-560$.

[5] K. N. Frederick, The Hopfian property for a class of fundamental groups, Comm. Pure Appl. Math. 16 (1963), 1-8.

[6] J. Hempel, Residual finiteness of surface groups, Proc. Amer. Math. Soc. 32 (1972), 323.

[7] W. Magnus, Beziehungen zwischen Gruppen und Idealen in einem speziellen Ring, Math. Ann. 111 (1935), no. 1, 259-280.

[8] W. S. Massey, Algebraic topology: An introduction, Harcourt, Brace \& World, Inc., New York, 1967.

[9] A. Reznikov, Crossing number and lower central series of a surface group, unpublished preprint, 1998.

[10] J. J. Rotman, An introduction to the theory of groups, Fourth edition, Springer, New York, 1995. 
Justin Malestein

Department of Mathematics

University of Chicago

5734 University Avenue

Chicago, IL 60637-1514

E-mail: justinm@math.uchicago.edu
Andrew Putman

Department of Mathematics

MIT, 2-306

77 Massachusetts Avenue

Cambridge, MA 02139-4307

E-mail: andyp@math.mit.edu 